\title{
Absorção de chumbo antrópico por populações de liquens do gênero Usnea em área industrial
}

\author{
Absorption of anthropogenic lead by populations of lichens of the genus Usnea in industrial area \\ Juliana Ferreira Soares', Róbson Ilha², Maria Amélia Zazycki³, Rodrigo Costa Carvalho Bernardes ${ }^{4}$, \\ Sérgio Roberto Mortari ${ }^{5}$, Noeli Júlia Schussler de Vasconcellos ${ }^{6}$ \\ 1,3 Mestranda no Programa de Pós Graduação em Engenharia de Processos, Universidade Federal de Santa Maria \\ 2 Mestre em Engenharia Agrícola, Universidade Federal de Santa Maria \\ ${ }^{4}$ Especialização em Engenharia de Segurança do Trabalho, Centro Universitário Franciscano \\ ${ }^{5}$ Professor adjunto, Centro Universitário Franciscano \\ ${ }^{6}$ Professora adjunto, Centro Universitário Franciscano
}

\begin{abstract}
Resumo
As emissões industriais de chumbo, oriundas, principalmente, de processos de fusões secundárias, provocam um aumento da concentração deste na atmosfera, degradando a qualidade do meio ambiente e, consequentemente, interferindo na saúde humana. O uso de bioindicadores tem sido uma importante ferramenta na avaliação da qualidade atmosférica, visto que estes organismos têm estreitas relações ecofisiológicas com a atmosfera e alto grau de acumulação de poluentes. O objetivo deste estudo foi quantificar a absorção de chumbo antrópico por populações de liquens do gênero Usnea nas proximidades de uma retificadora de baterias automotivas, localizada no município de Santa Maria - RS. O trabalho foi conduzido por meio da exposição de liquens do gênero Usnea, oriundos de área nativa, nas proximidades da empresa, durante o inverno e a primavera de 2010. A quantificação de chumbo foi realizada por espectrometria de emissão óptica. A análise quantitativa indicou altos níveis de chumbo absorvidos pela população de líquens, evidenciando que há poluição de origem atmosférica. Este resultado confirma a ineficácia das práticas de controle de emissão de poluentes.
\end{abstract}

Palavras-chaves: Bioindicadores, Qualidade do ar, Metais pesados.

\begin{abstract}
The industrial emissions of lead, from mainly mergers, causing a secondary increase in concentration of the atmosphere, degrading the quality of the environment and hence interfering with the human health. The use of bioindicators has been an important tool in the assessment of air quality, since these organisms have close relations with ecophysiological atmosphere and high degree of accumulation of pollutants. The aim of this study was to quantify the absorption of anthropogenic lead in populations of lichens of the genus Usnea near a grinding of automotive batteries, located in the municipality of Santa Maria - RS. The work was conducted by exposing lichens of the genus Usnea, coming from native area, nearby the company, during the winter and spring of 2010. Quantification of lead was performed by optical emission spectrometry. The quantitative analysis indicated high levels of lead absorbed by the lichen community, evidencing the origin of atmospheric pollution. This result confirms the ineffectiveness of measures to control the emission of pollutants.
\end{abstract}

Keywords: Bioindicators, Air quality, Heavy metals. 


\section{INTRODUÇÃO}

As emissões industriais de chumbo, oriundas, principalmente, de processos de fusões secundárias, provocam um aumento da concentração deste na atmosfera, degradando a qualidade do meio ambiente e, consequentemente, interferindo na saúde humana. Organismos vivos, como os liquens, os quais podem ser utilizados como bioindicadores de contaminação atmosférica por metais, surgem como um método a fim de estimar o impacto causado pelas indústrias, uma vez que possuem alto grau de acumulação de poluentes (Quiterio et al., 2006; Szczepaniak \& Biziuk, 2003; Leonardo et al., 2011). Este método além de ser fácil, é também econômico e permite avaliar a qualidade do ar em áreas extensas, além de fornecer informações rápidas e seguras sobre as ações dos poluentes na biota (Mariani et al., 2008; Teixeira \& Barbério, 2012).

O biomonitoramento é mundialmente conhecido como um método de análise experimental indireto na avaliação da qualidade ambiental, utilizando-se de organismos vivos que respondem ao estresse promovido por substâncias poluentes que se acumulam em seus tecidos, ocasionando modificações bioquímicas, fisiológicas e morfológicas. Esses organismos são geralmente denominados bioindicadores, uma vez que são resistentes e têm estreitas relações ecofisiológicas com a atmosfera, motivo pelo qual constituem uma ferramenta promissora para a bioindicação da poluição atmosférica (Villarouco et al., 2007; Teixeira \& Barbério, 2012; Cáceres, 1996).

Dentre os organismos bioindicadores, destacam-se os liquens, os quais são formados pela associação simbiótica entre fungos e algas. Essa associação forma uma estrutura sem raízes ou cutículas, dependendo principalmente da atmosfera para obtenção de nutrientes. Os liquens são os organismos mais estudados para avaliar a qualidade do ar, e têm sido descritos como um dos melhores bioindicadores de metais pesados na atmosfera, pois são capazes de acumular e tolerar níveis de metal muito acima de suas necessidades fisiológicas (Wolterbeek et al., 2003; Carneiro, 2004; Carreras \& Pignata, 2002). No estado de São Paulo e em três municípios da região metropolitana, por exemplo, os líquens têm sido utilizados para detectar a presença de metais pesados na atmosfera (Lisboa \& Kawano, 2007).

Para avaliar a bioacumulação dos poluentes, os liquens podem ser utilizados de duas maneiras, utilizando os organismos presentes na própria área de pesquisa, ou transplantados de uma área sem contaminação para o local potencialmente contaminado. A alta sensibilidade desses organismos aos poluentes pode ser mensurada por métodos de análise de extratos dos talos liquênicos submetidos à contaminação (Conti \& Cecchetti, 2001).

As fábricas reformadoras de baterias, por usarem processos e tecnologias obsoletas, são uma das principais fontes de emissão de chumbo para a atmosfera, causando um aumento da poluição ambiental principalmente nas áreas vizinhas e consequentemente acarretando danos à saúde humana, como alterações nos sistemas neurológico, hematológico, metabólico e cardiovascular (Quiterio et al., 2006).

Com isso, este trabalho teve como objetivo quantificar a absorção de chumbo antrópico por líquens do gênero Usnea nas proximidades de uma retificadora de baterias automotivas, localizada no Distrito Industrial do município de Santa Maria - RS.

\section{METODOLOGIA}

No presente estudo foram utilizadas populações de liquens do gênero Usnea como bioindicadores de acumulação para a quantificação de chumbo $(\mathrm{Pb})$ nas proximidades de uma retificadora de baterias automotivas. A área de estudo localiza-se no Distrito Industrial do município de Santa Maria, à aproximadamente quinhentos metros da BR 287 e de um dos maiores bairros da cidade. $\mathrm{O}$ município está localizado a uma latitude de $29^{\circ} 41^{\prime} 02^{\prime \prime} \mathrm{S}$ e longitude de $53^{\circ} 48^{\prime} 25^{\prime \prime} \mathrm{O}$, com altitude de 151 metros e apresenta clima subtropical úmido.

Os liquens foram obtidos de uma área nativa, situada no interior do município de Itaara - RS, próximo à cidade de Santa Maria, onde não há fontes potenciais de poluição atmosférica. De acordo com a figura 1, os liquens, juntamente com seus substratos, foram expostos em dois pontos nas proximidades da retificadora de baterias, sendo P1 em frente da empresa e P2 ao lado direito. Por não se ter conseguido acesso, não foi possível colocar amostras de liquens aos fundos e no lado esquerdo da retificadora. As amostras foram dispostas em triplicatas entre a vegetação local durante as estações de inverno e primavera de 2010. Após cada estação, os liquens eram removidos dos seus respectivos 
substratos, secados em estufa a $70^{\circ} \mathrm{C}$, triturados em Grall e preparados para a extração e quantificação do chumbo.

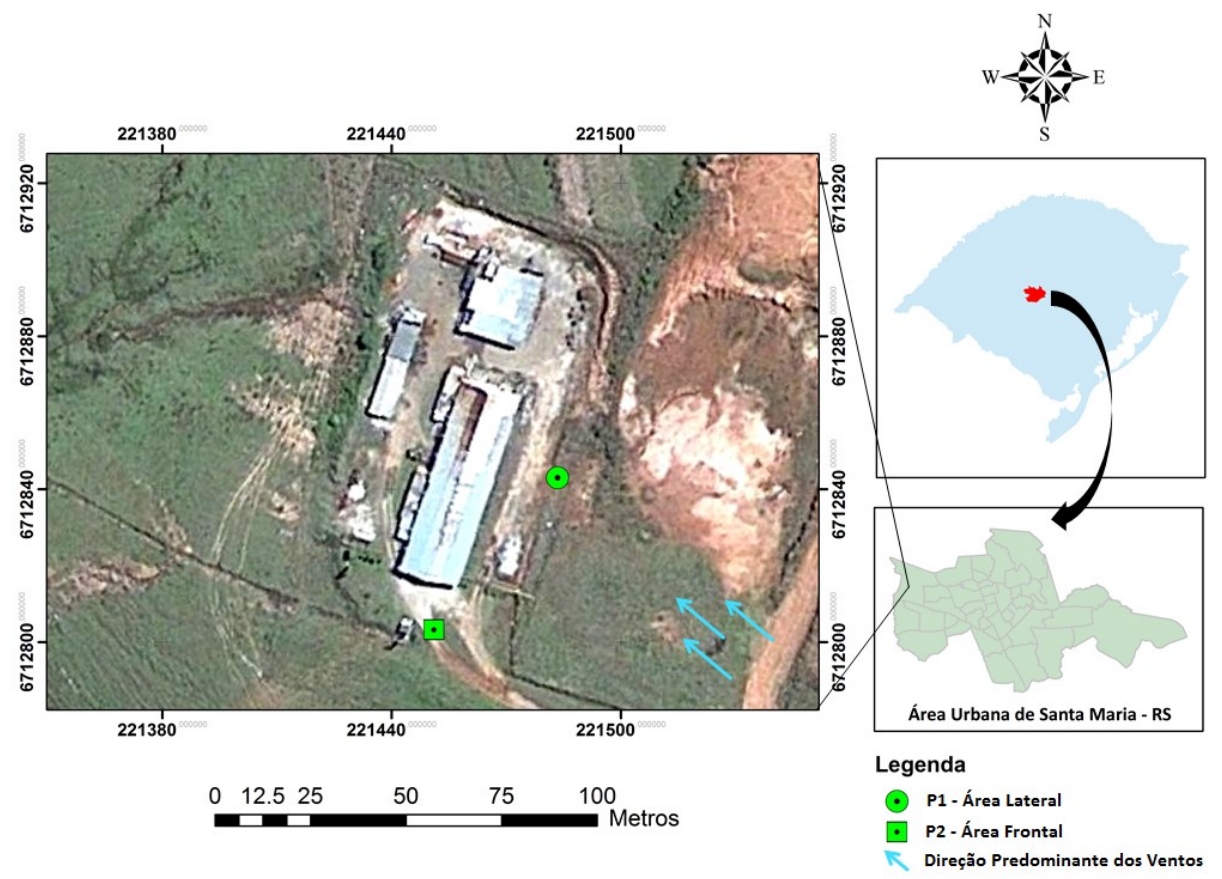

Figura 1. Localização dos pontos de exposição dos líquens na retificadora de baterias automotivas para quantificação de chumbo na atmosfera.

Para a determinação de $\mathrm{Pb}$ nos liquens foi utilizada a técnica de espectrometria de emissão óptica com plasma indutivamente acoplado (ICP OES), Spectro Analytical Instruments (modelo SpectroCiros CCD, equipado com uma câmara de nebulização duplo passo tipo scott e nebulizador pneumático do tipo croosflow). O equipamento foi calibrado com solução de calibração multielementar SPC Science 33 MS e os parâmetros operacionais utilizados seguiram as recomendações do fabricante, conforme mostra o Quadro 1.

As amostras dos extratos foram pesadas (200mg) em balança analítica e diluídas em água destilada e deionizada em coluna de troca iônica convencional e, posteriormente, purificada em sistema Milli-Q ${ }^{\circledR}$. As diluições foram realizadas em função das concentrações dos analitos nas amostras (10, 100 ou 1000 vezes).

Quadro 1. Parâmetros operacionais utilizados para as determinações por ICP OES.

\begin{tabular}{|l|c|}
\hline Potência (W) & 1300 \\
\hline Radiofreqüência (MHz) & 27 \\
\hline Vazão de Argônio Principal $\left(1 \mathrm{~min}^{-1}\right)$ & 14,0 \\
\hline Vazão de Argônio Auxiliar $\left(1 \mathrm{~min}^{-1}\right)$ & 1,0 \\
\hline Vazão de Nebulização $\left(1 \mathrm{~min}^{-1}\right)$ & 1,0 \\
\hline Nebulizador & Tipo Cross Flow \\
\hline
\end{tabular}




\section{RESULTADOS E DISCUSSÃO}

Os resultados da quantificação de chumbo presentes nos extratos liquens do gênero Usnea expostos em dois pontos próximos à retificadora de baterias automotivas, localizada no município de Santa Maria, durante as estações de inverno e primavera de 2010 estão apresentados na tabela 1. A tabela 2 mostra os dados meteorológicos do período de estudo obtidos pelo INMET.

Tabela 1. Valores médios de chumbo detectado nos extratos das populações de liquens do gênero Usnea expostas nas proximidades do distrito industrial de Santa Maria durante as estações de inverno e primavera de 2010.

\begin{tabular}{cccc}
\hline \multirow{2}{*}{ Ponto } & \multicolumn{3}{c}{ Concentração de Chumbo $\left(\boldsymbol{\mu g} \cdot \mathbf{g}^{-1}\right)$} \\
\cline { 2 - 4 } & Testemunha & Inverno & Primavera \\
\hline P1 (lateral) & $3,4586 \pm 0,0005$ & $393,97 \pm 0,003$ & $486,54 \pm 0,003$ \\
P2 (frontal) & & $147,80 \pm 0,005$ & $200,50 \pm 0,02$ \\
\hline
\end{tabular}

Tabela 2. Dados meteorológicos dos períodos de exposição dos líquens ao chumbo antrópico no Distrito Industrial de Santa Maria.

\begin{tabular}{ccc}
\hline Dados Meteorológicos & Inverno & Primavera \\
\hline Direção predominante dos ventos & \multicolumn{2}{c}{ Sudeste (SE) } \\
Velocidade média do vento $\left(\mathrm{m} \cdot \mathrm{s}^{-1}\right)$ & 2,07 & 2,18 \\
Precipitação $(\mathrm{mm})$ & 613,7 & 280,1 \\
Umidade relativa média do ar & 83,24 & 69,74 \\
\hline
\end{tabular}

Fonte: INMET.

A concentração de chumbo nos extratos dos liquens de origem nativa que foram transplantados para a área de estudo, ou seja, na área de potencial fonte de poluição, foi de 43 a 141 vezes maior que no extrato dos líquens testemunha. Este resultado permite dizer que a retificadora de baterias automotiva pode estar emitindo quantidades significantes de chumbo para a atmosfera. Foram detectadas concentrações de chumbo nos liquens entre 147,8 a 486,54 $\mu \mathrm{g}$. $\mathrm{g}^{-1}$, enquanto que no trabalho de Leonardo et al (2011), o qual quantificou a presença de chumbo em liquens expostos nas proximidades de uma empresa de estanho e chumbo, foram encontradas concentrações ainda maiores, entre 401 a $1083 \mu \mathrm{g}$. g-1.

$\mathrm{Na}$ figura 2 pode-se observar claramente que no ponto $\mathrm{P} 1$ foi detectada maior concentração de chumbo nos extratos dos liquens para ambos os períodos, e de um modo geral, todos os resultados de concentração foram muito maiores que a testemunha. Nota-se, também, que na primavera as concentrações nos pontos P1 e P2 foram maiores que no inverno.

A diferença de concentração de chumbo nos pontos $\mathrm{P} 1$ e $\mathrm{P} 2$ pode ser atribuída às condições das correntes eólicas e pelo fato de P1 localizar-se na entrada da retificadora de baterias, onde há maior fluxo de entrada e saída de veículos e mercadorias. O vento é um fator que pode influenciar no transporte e disposição das partículas de poluentes, como o chumbo (Quiterio et al., 2006). Isto pode ser observado no ponto P2, que apresentou concentrações mais baixas de chumbo, visto que este ponto esta localizado na direção oposta à direção predominante dos ventos (sudeste).

Os poluentes podem, então, estar sendo transportados para a direção sudeste, onde não foi 


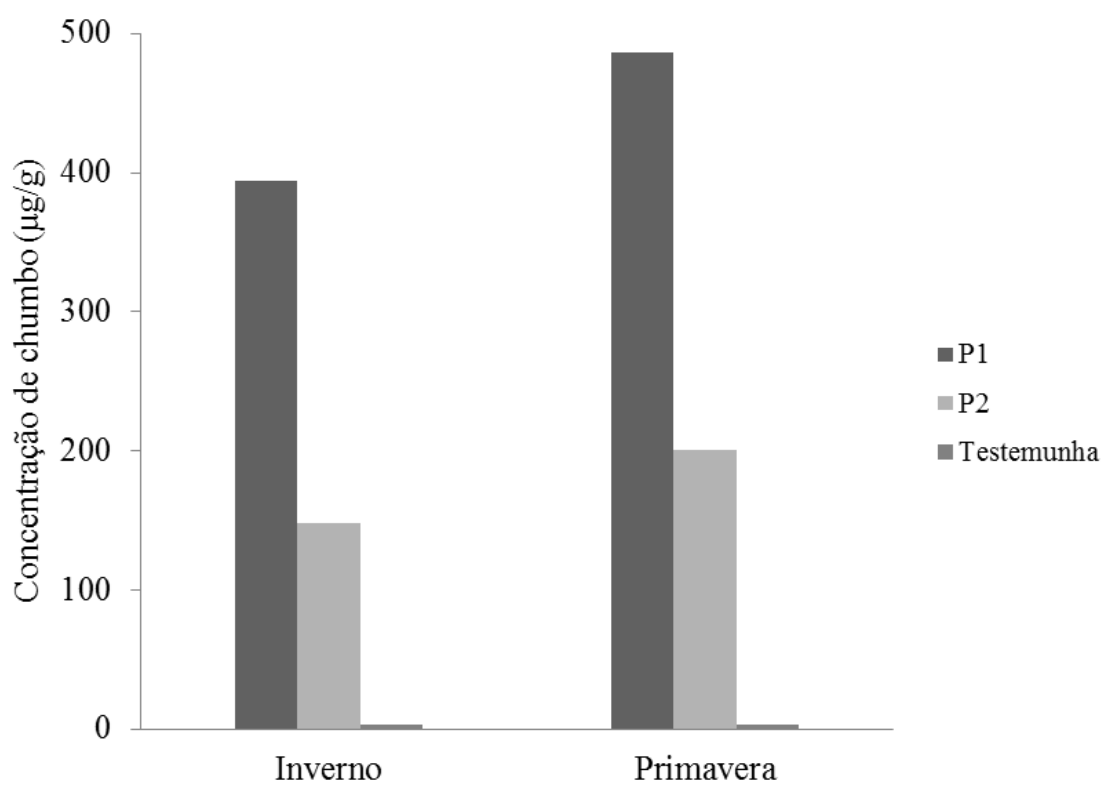

Figura 2. Concentração de chumbo detectado nos extratos dos liquens expostos em dois pontos no período de inverno e primavera e a concentração de chumbo na amostra testemunha.

possível ter acesso pra expor os liquens. A influência do vento na difusão dos poluentes foi detectada, também, no estudo desenvolvido por Leonardo et al. (2011), onde foi observado que as concentrações de metais, entre eles o chumbo, foi maior nos pontos onde a direção predominante dos ventos incidia.

No período de menor concentração de chumbo (inverno) os dados de precipitação e a umidade relativa do ar foram maiores. Isto pode ter ocorrido devido ao carregamento dos poluentes pela chuva e consequente deposição no solo.

\section{CONCLUSÃO}

A utilização de liquens do gênero Usnea como bioindicadores de poluição atmosférica por chumbo mostrou-se satisfatória, tendo em vista as elevadas quantidades de chumbo encontradas nos liquens expostos no local de estudo.

Os parâmetros climáticos foram fundamentais para avaliar o comportamento do transporte e deposição dos poluentes. As condições do vento e das precipitações mostraram-se estar diretamente relacionadas com as quantidades de chumbo encontradas em cada ponto estudado.

Os resultados obtidos no trabalho mostram a necessidade de adoção de medidas preventivas de controle da emissão de poluentes pela empresa e a ação das autoridades em fiscalizar estas fontes de poluição que afetam diretamente a saúde dos trabalhadores e da população que reside nas proximidades.

\section{REFERÊNCIAS}

CÁCERES, M. E. S. Biomonitoramento ativo e passivo da qualidade do ar atmosférico na cidade do Recife -PE. Recife, 1996. 77 p. Monografia (Graduação em Ciências Biológicas). Departamento de Ciências Biológicas, Universidade Federal de Pernambuco.

CARNEIRO, R. M. A. Bioindicadores vegetais de poluição atmosférica: uma contribuição para a saúde da comunidade. Ribeirão Preto, 2004. 169 p. Dissertação (Mestrado em Enfermagem em Saúde Pública) - Escola de Enfermagem de Ribeirão Preto, Universidade de São Paulo.

CARRERAS, H. A. \& PIGNATA, M. L. Biomonitoring of heavy metals and air quality in Cordoba City, Argentina, using transplanted lichens. Environmental Pollution, v. 117, p. 77-87, 2002. 
CONTI, M. E. \& CECCHETTI, G. Biological monitoring: lichens as bioindicators of air pollution assessment - a review. Environmental Pollution, v. 114, p. 471-492. 2001.

INMET - INSTITUTO NACIONAL DE METEOROLOGIA. Banco de Dados Meteorológicos para Ensino e Pesquisa - BDMEP. Disponível em: <http://www.inmet.gov.br/portal/index.php?r=bdmep/bdmep>. Acesso em: 20 de maio de 2014.

LEONARDO, L.; MAZILLI, B. P.; DAMATTO, S. R.; SAIKI, M.; OLIVEIRA, S. M. B. de. Assessment of atmospheric pollution in the vicinity of a tin and lead industry using lichen species Canoparmelia texana. Journal of Environmental Radioactivity, v. 102, p. 906-910, 2011.

LISBOA, H. de M. \& KAWANO, M. Monitoramento de Poluentes atmosféricos. In: LISBOA, H. DE M. Controle da Poluição Atmosférica. Montreal, v. 1, p. 1-73, 2007.

MARIANI, R. L.; JORGE, M. P. M.; PEREIRA, S. S. Caracterização da qualidade do ar em são José dos Campos-SP, utilizando Tradescantia Pallida (TRAD-MCN). Geochimica Brasiliensis, v. 22, n. 1, p. 027-033, 2008.

QUITERIO, S. L.; MOREIRA, F. R.; SILVA, C. R. S. da; ARBILLA, G.; ARAÚJO, U. C.; MATTOS, R. de C. O. da C. Avaliação da poluição ambiental causada por particulado de chumbo emitido por uma reformadora de baterias na cidade do Rio de Janeiro, Brasil. Cadernos de Saúde Pública, v. 22, n. 9, p. 1817-1823, 2006.

SZCZEPANIAK, K. \& BIZIUK, M. Aspects of the biomonitoring studies using mosses and lichens as indicators of metal pollution. Environmental Research, v. 93, p. 221-230, 2003.

TEIXEIRA, M. C. V. \& BARBÉRIO, A. Biomonitoramento do ar com Tradescantia pallida (Rose) D. R. Hunt var purpurea Boom (Commelinaceae). Revista Ambiente \& Água - An Interdisciplinary Journal of Applied Science, v. 7, n.3, p. 279-292, 2012.

VILlAROUCO, F. M. de O.; FREIRE, E. V. C.; PEREIRA, E. C. G.; PÉREZ, R. E. U. Análise do líquen Cladonia Verticillaris (RADDI) FR., em condições de laboratório sob efeito do estireno e ciclohexano. Interciência, v. 32, n. 4, p. 242-246, 2007.

WOLTERBEEK, H. T.; GARTY, J.; REIS, M. A.; FREITAS, M. C. Biomonitors in use: lichens and metal air pollution. In: MARKERT, B.A.; BREURE, A.M.; ZECHMEISTER, H.G. (Editores), Bioindicators and biomonitors. Elsevier Science Ltd., v. 11, p. 377-419, 2003. 